UDC 539.6

DOI: $10.20333 / 2500136-2021-2-83$

\title{
New aptamer-based approaches for the targeting of cancer associated fibroblast in NSCLC
}

G. Ciccone ${ }^{1}$, M. L. Ibba ${ }^{2}$, G. Coppola 2 , G. Petrillo², S. Catuogno ${ }^{1}$, C. L. Esposito ${ }^{1}$

${ }^{1}$ Institute Exper imental Endocrinology and Oncology "Gaetano Salvatore" (IEOS), National Research Council (CNR), Naples 80145, Italy

${ }^{2}$ Department of Molecular Medicine and Medical Biotechnology, "Federico II" University of Naples, Naples 80131, Italy

The aim of the research. Non-small cell lung cancer (NSCLC) represents about $80 \%$ of all lung cancer cases and is often associated with drug resistance, relapses and a poor prognosis. Therefore, the identification of effective therapeutic strategies represents a crucial challenge in oncology. A key limit of conventional anticancer treatments is that they do not target the permissive tumor microenvironment, of which key components are cancer-associated fibroblasts (CAFs). It has been shown that CAFs are able to regulate malignant progression and drug resistance. However, a detailed characterization of CAF profile and the targeting of their pro-tumor effects still remain an ambitious challenge and have a primary importance for the identification of new effective therapies.

Material and methods. We aimed to develop innovative strategies based on nucleic acid aptamers to address these fundamental issues. Firstly, we applied an aptamer conjugate (named Gint4.T-STAT3), containing a STAT3 siRNA linked to an aptamer binding and inhibiting the PDGFR $\beta$, to specifically silence STAT3 reported as a fundamental player in the cross-talk between CAFs and epithelial NSCLC cells.

Results. We demonstrated that this molecule effectively delivers STAT3 siRNA in NSCLC cells, blocking CAF-induced cell growth and migration in both continous and primary NSCLC coltures. In addition, in order to address CAF specific targeting and profiling, we developed an innovative differential cell-SELEX approach by using primary NSCLC CAFs as selection target. Such a strategy allowed the isolation of different aptamers discriminating NSCLC CAFs from normal lung fibroblasts. The analyses of aptamer specificity and functionality is curently ongoing.

Conclusion. Our data represent the first ever attempt in CAF targeting using aptamer-based drugs, and can open innovative horizons in the current therapeutic approaches forNSCLC.

Key words: aptamers, cancer cell, fibroblast, non-small cell lung cancer.

Conflict of interest. The authors declare the absence of obvious and potential conflicts of interest associated with the publication of this article.

Citation: Ciccone G, Ibba ML, Coppola G, Petrillo G, Catuogno S, Esposito CL. New aptamer-based approaches for the targeting of cancer associated fibroblast in NSCLC. Siberian Medical Review. 2021; (2):83. DOI: 10.20333/2500136-2021-2-83

\footnotetext{
Author information

G. Ciccone, Institute Experimental Endocrinology and Oncology "Gaetano Salvatore" (IEOS), National Research Council (CNR), 80145 Naples, Italy

M. L. Ibba, Department of Molecular Medicine and Medical Biotechnology, "Federico II" University of Naples, 80131 Naples, Italy

G. Coppola, Department of Molecular Medicine and Medical Biotechnology, "Federico II" University of Naples, 80131 Naples, Italy
}

G. Petrillo, Department of Molecular Medicine and Medical Biotechnology, "Federico II" University of Naples, 80131 Naples, Italy

Silvia Catuogno, PhD, Istituto di Endocrinologia ed Oncologia Sperimentale, Consiglio Nazionale delle Ricerche (CNR), 80100, Naples, Italy; e-mail: s.catuogno@ieos.cnr.it

Carla L. Esposito, PhD, Istituto di Endocrinologia ed Oncologia Sperimentale, Consiglio Nazionale delle Ricerche (CNR), 80100, Naples, Italy; e-mail: c.esposito@ieos.cnr.it

Received 01 March 2021 Revision Received 18 March 2021 Accepted 31 March 2021 